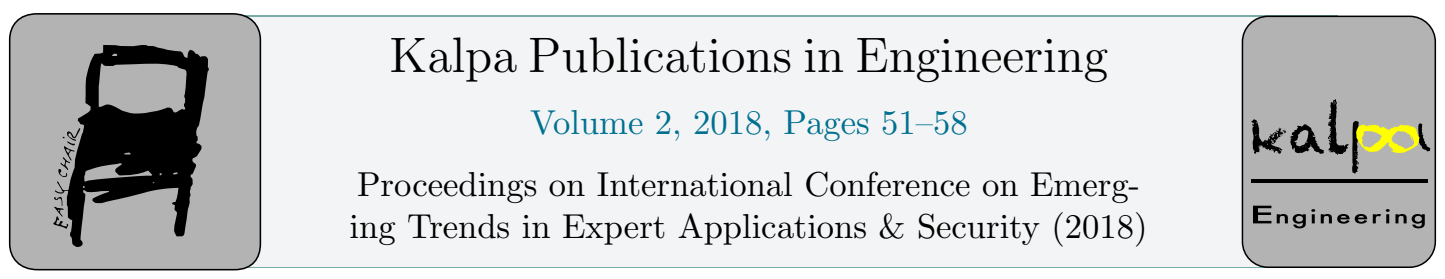

\title{
Denoising of ECG Signals Using FIR \& IIR Filter: A Performance Analysis
}

\author{
Ms. Chhavi Saxena ${ }^{1}$, Mr. Vivek Upadhyaya ${ }^{2}$, Dr. Hemant Kumar Gupta ${ }^{3}$, \\ Dr. Avinash Sharma ${ }^{4}$ \\ ${ }^{1,2}$ Arya College of Engineering \& I.T. Jaipur, Rajasthan, India \\ ${ }^{3}$ Poornima College of Engineering, Jaipur, Rajasthan, India \\ ${ }^{4}$ Maharishi Markandeshwar University, Ambala, Haryana \\ chhavisaxena_81@rediffmail.com, vivekpragya@gmail.com, \\ hkg_1480@̄rediffmail.com,sh_avinash@yahoo.com
}

\begin{abstract}
Electrocardiogram (ECG) signal is a bio-electrical activity of the heart. It is a common routine and important cardiac diagnostic tool where in electrical signals are measured and recorded to know the functional status of heart, but ECG signal can be distorted with noise as, various artifacts corrupt the original ECG signal and reduces it quality. Therefore, there is a need to remove such artifacts from the original signal and improve its quality for better interpretation. Digital filters are used to remove noise error from the low frequency ECG signal and improve the accuracy the signal. Noise can be any interference due to motion artifacts or due to power equipment that are present where ECG had been taken. Thus, ECG signal processing has become a prevalent and effective tool for research and clinical practices. This paper presents the comparative analysis of FIR and IIR filters and their performances from the ECG signal for proper understanding and display of the ECG signal.
\end{abstract}

\section{Introduction}

Heart related diseases are among the major causes of human deaths all over the world. Therefore, to understand the physiological and functional status of heart, an efficient tools and methods for effective diagnosis of the cardiac disease is required. Electrocardiography (ECG) is a type of tool on which it records the all heart electric activity. In digital world computerized ECG analysis is used due to high reliable feature for diagnosis the heart related diseases. The ECG recordings are received with the help of electrodes, which are placed on the patient's chest and limb. Most of the signal taken by the chest and limb combination is affected from the different types of artifacts like Power line interference, Baseline Drift, Motion artifacts, 
Electrode contact noise, and Instrumentation noise caused by electronic devices. Some noises are produced by electric main power system, during the time of the recording and monitoring of the ECG signals. In the whole system some noise has low frequency, which are producing some problem form ECG i.e. mobile phone. The frequency is measured in cycle/second or in "Hertz". For example the electric power used in daily life is $50 \mathrm{~Hz}$ in India [3].

In this paper, the main aim is to eliminate the noises of the electrocardiogram (ECG) using FIR and IIR filters. Because of Baseline noise interference, it becomes difficult to analyze the ECG records either manually or by automatic means.

\section{Digital Filters}

Digital filter is a arithmetical algorithm approach, which are implemented in hard ware and software. In this type of filter use a digital input to produce a digital output signal for achieving a filter objective. Two main purpose to use the digital filter as discussed as following : (i) Spilt the signal which has been combined and (ii) restorations of signal that have been distorted in some way signal separations needed when signal has been contaminated with interference, noise, or other signal. Filters may be:

- Linear or non-linear.

- Time-invariant or time-variant.

- Causal or non-causal Analog or digital.

- Discrete-time (sampled) or continuous-time.

- Passive or active type of continuous-time filter.

- Infinite impulse response (IIR) or finite impulse response (FIR) type of discrete-time or digital filter.

\section{Methodology}

Filter put into practice either as a program in the software or as a circuit in hardware. MATLAB is a important tool for our simulation purpose to obtain the convincible results. MATLAB has some attractive feature over the conventional computer languages for technical problem solving. As a below [6].

1. Ease of use.

2. Platform independence.

3. Predefined functions.

4. Device-independent plotting.

5. Graphical user interface.

6. MATLAB compiler Ease of use.

Firstly, the ECG signals are loaded from the database into the MATLAB tool for the execution procedure then these loaded signals are combined with the simulated signal. After that the ECG signal and noise are added. This added signal are put into examine procedure in time domain and the suitable design parameters for different digital filters. 


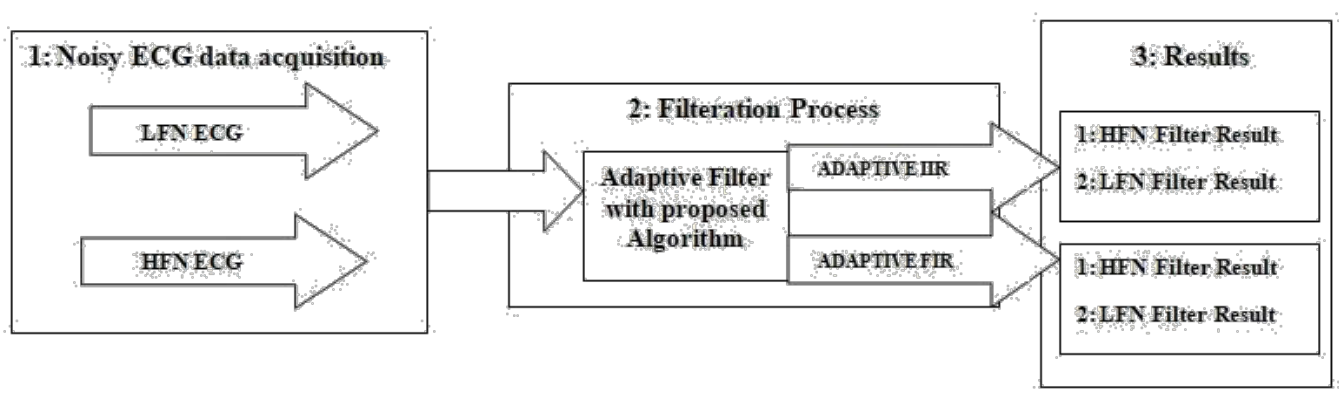

Figure 1 Step Wise Methodology For Filteration Process

\section{Algorithm Used}

The proposed algorithm is a great solution of the estimation problem with a least-squares cost function. Some algorithm like LMS adaption algorithm has slow convergence property due to which it is not able to work on $\mathrm{s}$ fast varying signal and not be suitable to observe the accurate value of the step size $\mu$. To minimize the problem a new algorithm is proposed. This method is mostly used in noise cancellation and real time system because it has a fast convergence and high magnitude as compare to the other algorithms. In which it used the information of the input data, it revert back the developed information when the proposed method is introduce on the spot. The arrival new data is used to calculate the tap weight vector at time n-1 and updated vector at time $n$.

For 1 to $\mathrm{n}=$ Final

i. Get $x[n], y[n]$

ii. Get $e[n]=x[n]-h^{\prime}[n-1] y[n]$

iii. Calculate Gain vector

iv. Update the filter Parameters

$$
K[n]=\frac{P[n-1] y[n]}{\lambda+y[n] P[n-1] y[n]}
$$

$$
h[n]=h[n-1]+k[n] e[n]
$$

v. Update the P matrix

end

$$
P[n]=\frac{1}{\lambda}(P[n-1]-K[n] y[n] P[n-1])
$$

\section{Results}

This paper compare the performance behaviors both FIR filter and IIR filter for a better simulation result by using MATLAB tool with and without noise on the various parameters. 


\subsection{Noise Removal Results For FIR \& IIR Filters}

The main motive of this paper to verify the proposed algorithm, In which it filtered the all ECG signals and examine the error of the filter and signal. In this paper we used the MATLAB software to evaluate the performance of the suggested filter, which are two types of filter.

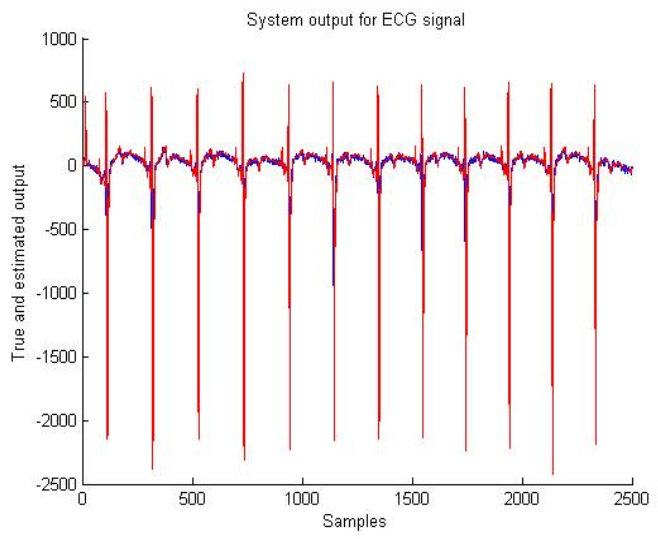

Figure 2 System Output of FIR Filter (Without Noise in ECG Signal)

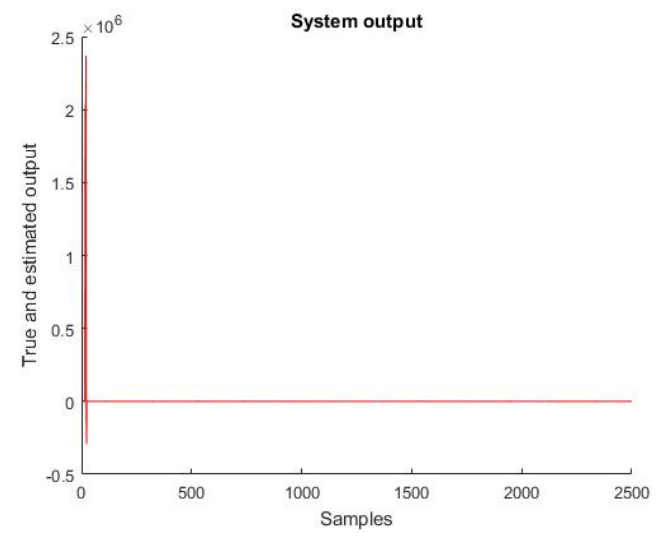

Figure 4 System Output of IIR Filter (Without Noise in ECG Signal)

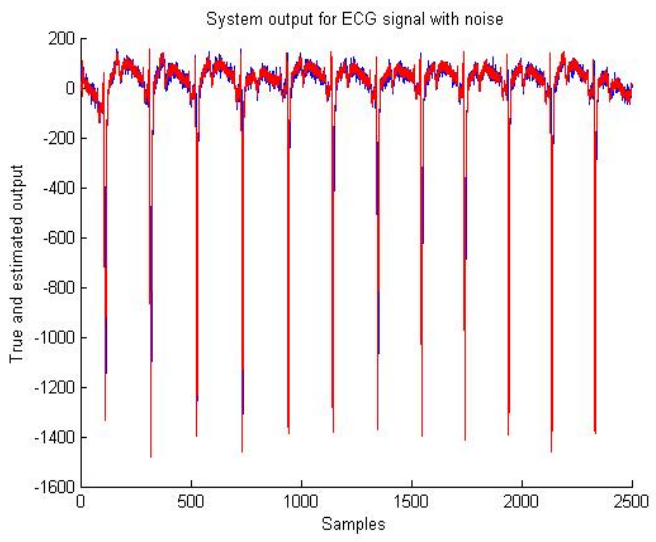

Figure 3 System Output of FIR Filter (With Noise in ECG Signal)

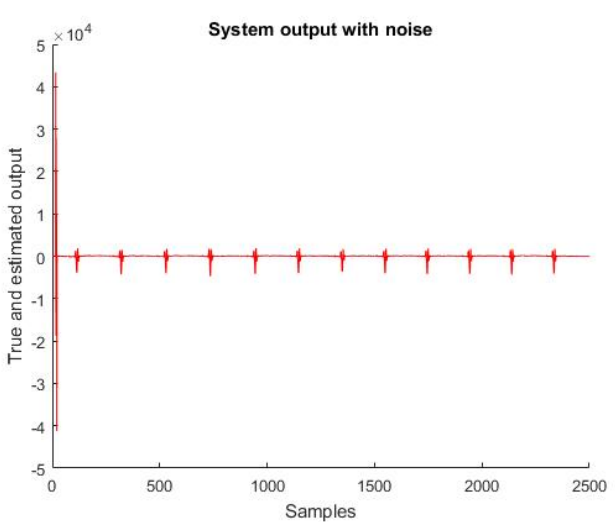

Figure 5 System Output of IIR Filter (With Noise in ECG Signal) 


\subsection{Error Calculation Results For FIR \& IIR Filters}

Figure 6,7,8,9 shows the error curve which are plotted using MATLAB respectively for FIR and IIR filters. A logarithmic scale is used for the calculation \& plotting of curves as it can show minor difference too in the spectrograms. By plot we can see easily that the high frequency components are suppressed much with the help of FIR rather than IIR filter.

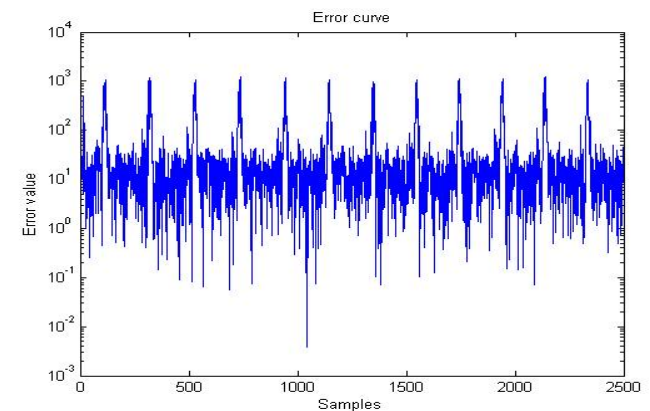

Figure 6 Error curve for FIR Filter (Without Noise in ECG signal)

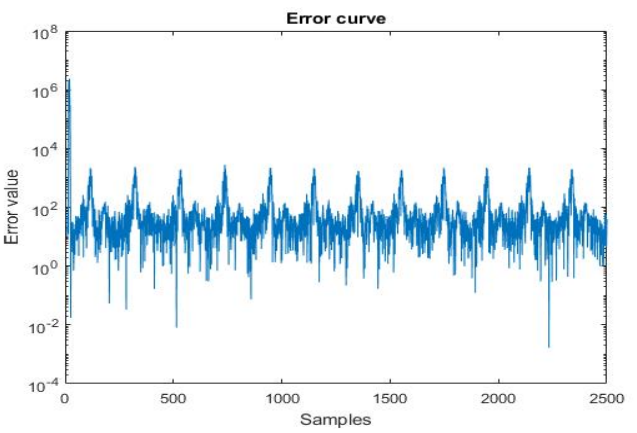

Figure 8 Error curve for IIR Filter (Without Noise in ECG signal)

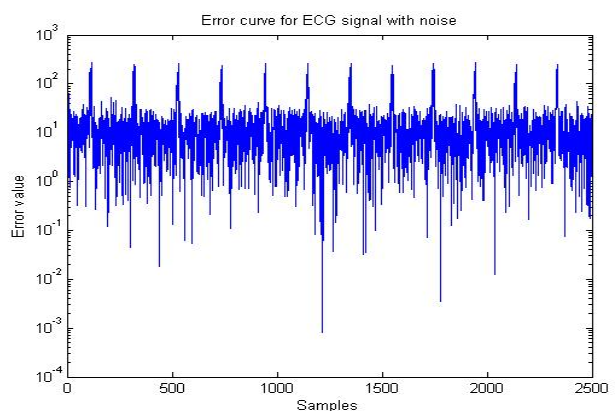

Figure 7 Error Curve for FIR Filter (With Noise in ECG Signal)

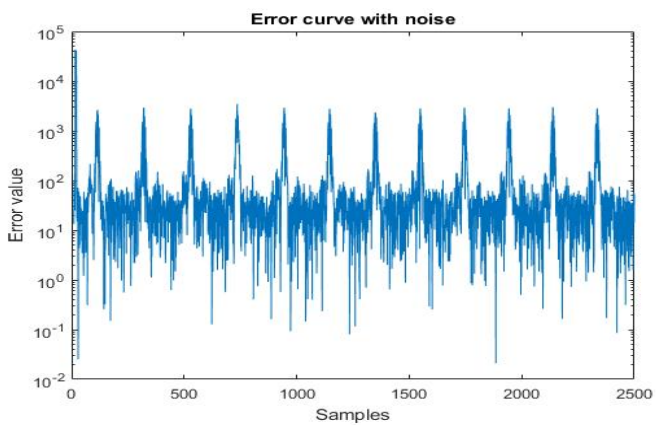

Figure 9 Error Curve for IIR filter (With Noise in ECG Signal)

\subsection{Comparison of the Filter Weights and Estimated Weights}

As a shown in the figure it shows a big comparison between the filter weight values and calculated weighted values of the FIR filter and IIR filter. As given following observation IIR filter have low order as compare to FIR filter, because of output of the FIR filter is calculated by the current and past input, but it not use the previous value of the output but not previous values of the output. It gives a poor practical result. Instead, IIR filter is recursive filter. It uses the previous output and input values then it give a good results. 


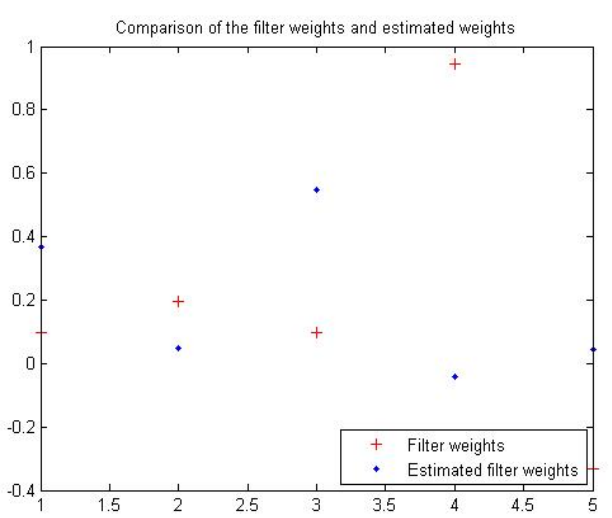

Figure 10 Comparison of FIR Filter weights \& estimated weights (Without Noise in ECG signal)

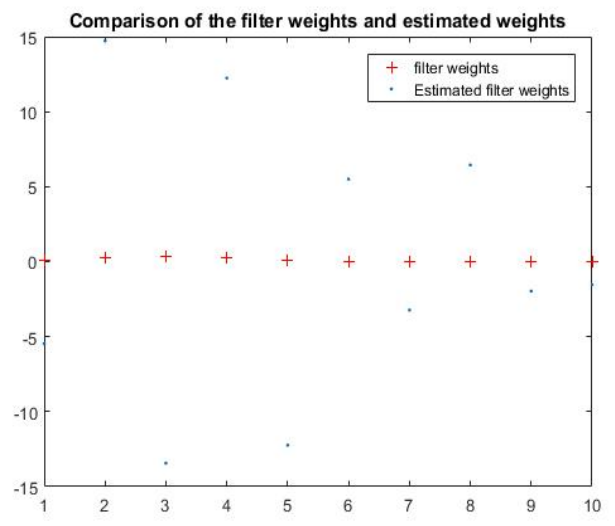

Figure 12 Comparison of IIR Filter weights \& estimated weights (Without Noise in ECG signal)

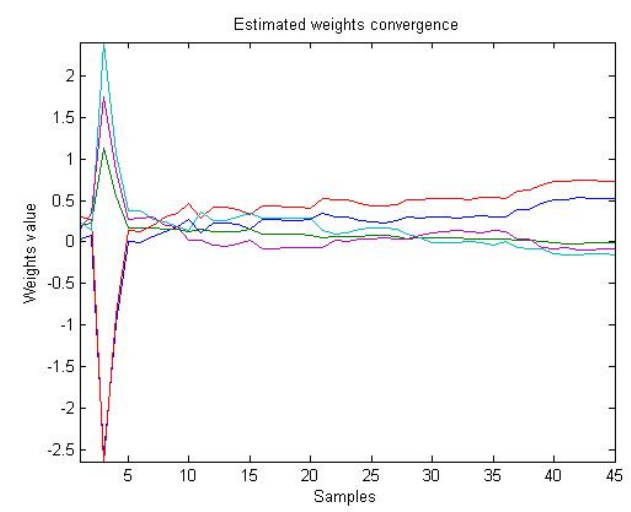

Figure 14 Estimated curve of FIR filter with noise

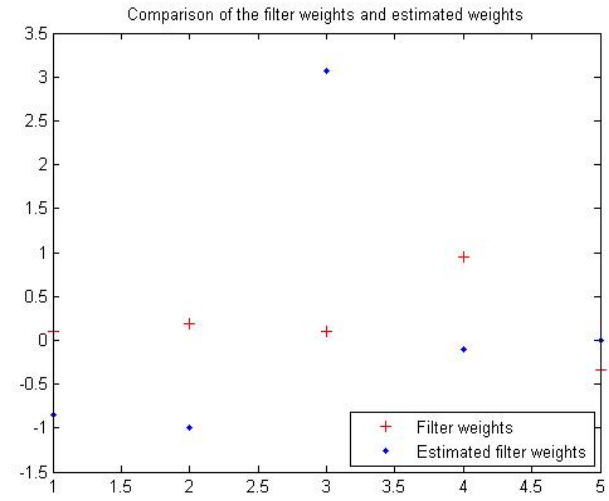

Figure 11 Comparison of FIR Filter weights \& estimated weights (With Noise in ECG signal)

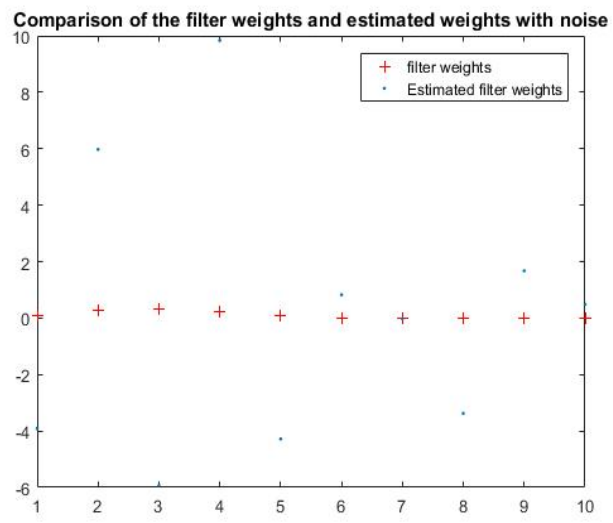

Figure 13 Comparison of IIR Filter weights \& estimated weights (With Noise in ECG signal)

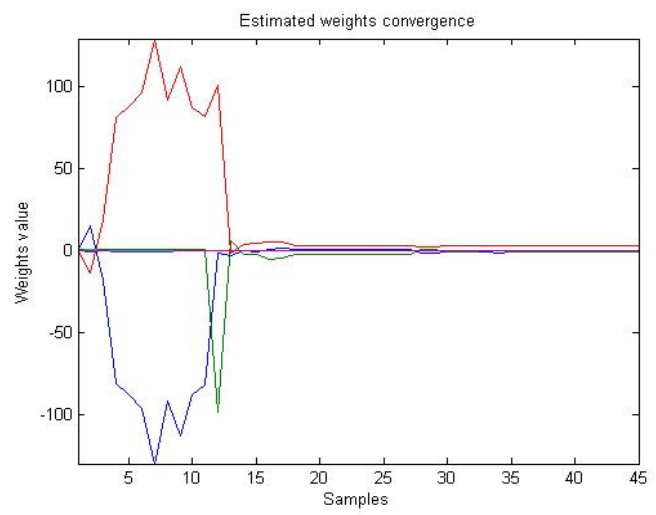

Figure 15 Estimated curve of FIR filter with noise 


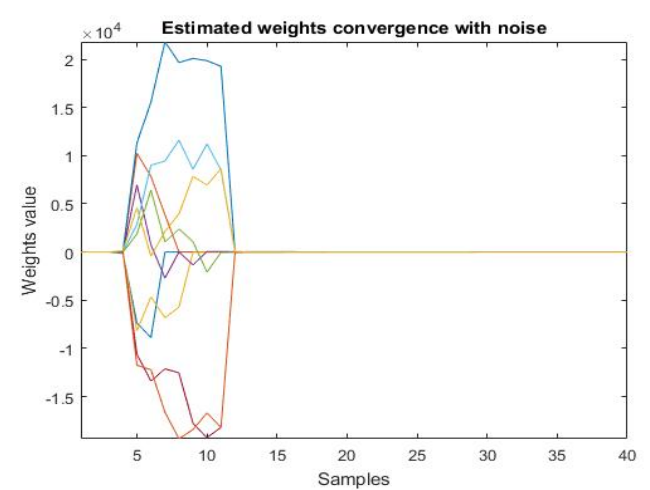

Figure 16 Estimated weight convergence of IIR filter with noise

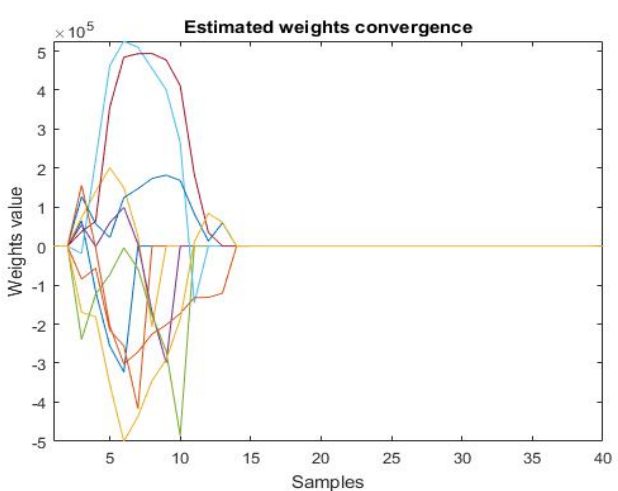

Figure 17 Estimated weight convergence of IIR without noise

\section{Conclusion}

Electrocardiogram is the commonly used signal in the field of biomedical. ECG signal is corrupted by different types of noises as a High Frequency Noise \& Low Frequency Noise. The algorithm which is used in this paper can improve the performance of filtering for these types of signals. In this paper FIR and IIR filter used to remove the High Frequency \& Low Frequency noise. The output of the FIR filter is very near about the desired value \& has small removal of High Frequency Noise \& Low Frequency Noise while the output which we get through IIR filter has less value than the desired output. Another important conclusion which we get through the observation is this that the standard deviation of IIR filter is much closer to the desired output \& the standard deviation of the FIR filter output with high frequency noise have less value than the IIR filter. The results which are given by FIR filter for proposed algorithm have much accurate results compared with IIR filter.

\section{Scope \& Future Work}

The objective of this paper is to analyze and compared different filters for ECG Signals. The results show that FIR filter is more stable than IIR filters. Too remove noise with the FIR filter is a best option as compare to IIR digital filter. In this paper, only FIR and IIR Filter is compared on two parameters, they can analyze on more parameters in future, further the results can be compared with other Filters.

\section{References}

[1] R.S Khandpur "Biomedical instrumentation hand book), $11^{\text {th }}$ reprint 2008 Tata McGraw -Hill publication company Limited New Delhi. ISBN-13: 978-0-07-0473355-3.

[2] Stephen J. chapman "MATLAB programming for engineers" $3^{\text {rd }}$ Reprint Edition 2003 by Thomson asia Pte Ltd., Singapore ISBN: 981-240-606-9. 
[3] Malindi, P.(2002) "Cancelling power line interference in electrophysiological signals". ECT Research Journal, 2.

[4] Widrow B. and Hoff M.E. (1960), "Adaptive switching circuits", In IREWESCON Convention Record, pp. 96-104, New York.

[5] Zhang Jiashu, Tai Heng-Ming, "Adaptive Noise Cancellation Algorithm for Speech Processing", IEEE Transactions, pp 2489-2492, 2007.

[6] Jigram H. Shah, Jay M. Joshi, "Digital signal processing” University science press laxmi publication company Limited New Delhi.

[7] Suzanna M. M. Martens , Massimo Mischi, S. Guid Oei, 'An Improved Adaptive Power Line Interference Canceller for Electrocardiography' IEEE transactions on biomedical engineering, vol. 53, no. 11, November 2006.

[8] Syed Zahurul Islam, Syed Zahidul Islam, Razali Jidin, Mohd. Alauddin Mohd. Ali, "Performance Study of Adaptive Filtering Algorithms for Noise Cancellation of ECG Signal", IEEE 2009.

[9] Kevin Buckley., " ECE5251 Biomedical Signal Processing", 2009

[10] Kiyoshi Nishikawa and Hitoshi Kiya, "Noval Implementation Technique of RLS Algorithm for Improving Throughtput of Adaptive Filters, 1999.

[11] ALI H. SAYED, "Adaptive Filters",2008

[12] Hayes, Monson H., "Statistical Digital Signal Processing and Modeling", John Wiley and Sons, inc., 1996.

[13] Chandra, Shanti, and Ambalika Sharma. "A computationally efficient approach for ECG signal denoising and data compression." In Control and System Graduate Research Colloquium (ICSGRC), 2017 IEEE 8th, pp. 22-27. IEEE, 2017.

[14] Hashemi, Abdolkarim, Masoomeh Rahimpour, and Mahmoud Reza Merati. "Dynamic Gaussian filter for muscle noise reduction in ECG signal." In Electrical Engineering (ICEE), 2015 23rd Iranian Conference on, pp. 120-124. IEEE, 2015.

[15] Lastre-Domínguez, Carlos, Yuriy S. Shmaliy, Oscar Ibarra-Manzano, and Luis J. MoralesMendoza. "Unbiased FIR denoising of ECG signals." In Electrical Engineering, Computing Science and Automatic Control (CCE), 2017 14th International Conference on, pp. 1-6. IEEE, 2017.

[16] Hesar, Hamed Danandeh, and Maryam Mohebbi. "ECG denoising using marginalized particle extended kalman filter with an automatic particle weighting strategy." IEEE journal of biomedical and health informatics 21, no. 3 (2017): 635-644. 